\title{
Analysis of the Strong and Weak Monotonic External Stability of the Resonance in a Perturbed Dynamical System
}

\author{
VLADISLAV V. LYUBIMOV \\ Samara National Research University, \\ 34, Moskovskoe shosse, Samara, 443086 \\ RUSSIA
}

\begin{abstract}
A perturbed dynamical system involving two ordinary differential equations is under review. Whereupon, the differential equation for determining the fast phase contains the ratio of the two frequencies. When these frequencies coincide for a long time, a resonance is implemented in this system. The aim of this paper is to obtain the conditions of monotonic external stability and instability of this resonance. The sufficient conditions for the external stability and instability of the resonance defined in this paper assume that the signs of the analyzed derivatives remain unchanged in the non-resonant section of the change in the independent variable. This paper gives a new classification of the phenomenon of external stability of resonance, which includes weak, linear, and strong stability. It should be noted that the conditions of monotonic external stability and instability of the resonance presented in this paper can be used in various scientific and technological problems, in which resonances are observed. Particularly, the concluding part of the paper considers the application of the results obtained within the framework of the problem of the perturbed motion of a rigid body relative to a fixed point.
\end{abstract}

Keywords: dynamical system, stability condition, equations, sign-constant derivative, resonance, rigid body.

Received: February 28, 2021. Revised: September 14, 2021. Accepted: September 25, 2021. Published: October 1,

2021.

\section{Introduction}

$\mathrm{T}$ he modern theory of differential equations traditionally uses the concepts of strong and weak stability of solutions. Particularly, publications [1-2] investigate the strong and weak-strong stability of solutions in various systems of equations describing the cross-diffusion phenomenon. Moreover, when solving the equations of mathematical physics, the concepts of strong and weak resonances are used. Thus, the papers [3-4] study strong and weak resonances obtained from delay-induced double Hopf bifurcations in systems of differential equations with delay. Let us note that the traditional aspect of studying the behavior of boundary cycles is the study of their stability.

The papers [5-8] describe methods for analyzing the stability and instability of boundary cycles in various nonlinear systems of differential equations. Similar to boundary cycles, resonances also include stability or instability properties in nonlinear systems of equations. In this case, the external and internal stability of the resonances are separated. The internal stability of resonances is considered, for example, in papers
[9-11]. Typically, the stability of oscillations in mechanical coupled oscillators is analyzed in this case. The concept of external stability of resonances with regard to differential systems with fast and slow variables was first described by Yu.A. Sadovy [12-13]. At the same time, it was assumed that the external stability of resonances should be understood as such behavior of the slow variables of the system beyond the asymptotically small resonance zone, in which the resonance is an attractor. Later, the paper [14] defines a more strict concept of the external stability of the resonance for a nonlinear system of equations of motion with slow and fast variables. Moreover, the use of the second Lyapunov method allowed obtaining the conditions for the external stability of the resonance [14]. The papers [15-16] present the results of the application of this method in the study of the external stability of the resonance in various problems of space flight dynamics.

It is important to note that the method for studying the external stability of the resonance is based on the analysis of the sign of the first derivative of the Lyapunov function.

This method does not allow analyzing the convexity of the function of the resonant frequency ratio as a function of the motion time. This paper proposes to analyze the monotonic external stability of the resonance, taking into account the signs of the first and second derivatives of the resonant 
frequency ratio. This result was achieved under the assumption that the right-hand sides are sign-constant, continuous and differentiable functions over the entire non-resonance time interval of motion. The aim of the paper is to obtain the conditions of monotonic external stability and instability of the resonance under consideration in a nonlinear perturbed twofrequency system of differential equations. Moreover, a joint analysis of the signs of the first and second derivatives of the resonant frequency ratio will allow categorizing three types of monotonic external stability of the resonance, i.e. "strong," "weak," and "linear." The concluding part of the paper contains an example of the application of this method within the framework of the problem of the perturbed motion of a rigid body relative to a fixed point.

\section{MATH Mathematical Model}

\subsection{Perturbed dynamical system}

Let us consider a system of differential equations:

$$
\begin{aligned}
& \frac{d \omega}{d t}=\mu \frac{f(\omega)}{\Delta(\omega)}, \\
& \frac{d \theta}{d t}=\Delta(\omega) .
\end{aligned}
$$

Here, $\mu$ is a small parameter that characterizes the value of the perturbing function, $f(\omega) ; \omega$ and $\theta$ are slow and fast variables, respectively; $f(\omega), \Delta(\omega)$ are known functions.

Let us call the function $\Delta(\omega)$ the resonant ratio of two frequencies $\quad \omega_{1}(\omega), \quad \omega_{2}(\omega) . \quad$ The equality $\Delta(\omega)=\omega_{1}(\omega)-\omega_{2}(\omega, c)$ will be true. Here, $c$ is a positive parameter. When an equality $\Delta\left(\omega_{1}(\omega), \omega_{2}(\omega)\right) \approx 0$ is satisfied in the system of equations (1)-(2), a resonance may occur. The size of the resonant range of values $\Delta(\omega)$, is characterized by size of the order $\sqrt{\varepsilon}$. Let us reduce our attention to the study of non-resonant areas of frequency ratio variation $\Delta(\omega)$.

Remark: It should be noted here that from the point of view of mathematics, the equation (1) should be considered independently of the equation (2). In what follows, the equations (1) and (2) are referred to as a system, assuming that equation (1) should be considered independently of the equation (2).

The functions $f(\omega(t)), \Delta(\omega(t))$ will be sign-constant, continuous, and differentiable on the non-resonant segment $t \in\left[t_{0}, t_{\mathrm{r}}\right)$ under consideration. Here, $t_{\mathrm{o}}$ is the initial value of the time $t$, and $t_{\mathrm{r}}$ is the value of the time when the value $\Delta$ reaches the resonant area $\Delta(\omega) \approx 0$. Moreover, the derivatives $\frac{d \Delta}{d t}$ and $\frac{d^{2} \Delta}{d t^{2}}$ are also sign-constant on the non-resonant segment $t \in\left[t_{\mathrm{o}}, t_{\mathrm{r}}\right)$ under consideration.

\subsection{Strong, weak, and linear external stability of the resonance}

First of all, let us introduce several concepts.

Definition 1. The linear monotonic external stability of an individual resonance, $\Delta \approx 0$, is understood as a monotonous convergence $\Delta$, to a small resonant area that occurs in a given non-resonant area, which is adjacent to the resonance by means of linear dependence $\Delta(t)$.

Definition 2. The weak nonlinear or simple-weak monotonic external stability of an individual resonance, $\Delta \approx 0$, is understood as a monotonous convergence $\Delta$ to a small resonant area that occurs in a given non-resonant area, which is adjacent to the resonance for a longer time interval than with linear external stability.

Definition 3. The strong nonlinear or simple-strong monotonic external stability of an individual resonance $\Delta \approx 0$ is understood as a monotonous convergence $\Delta$ to a small resonant area that occurs in a given non-resonant area, which is adjacent to the resonance for a shorter time interval than with linear external stability.

Remark: The initial values $\Delta(t), \frac{\mathrm{d} \Delta}{\mathrm{dt}}$, and $\frac{\mathrm{d}^{2} \Delta}{\mathrm{dt}^{2}}$ (whereupon, $\frac{\mathrm{d}^{2} \Delta}{\mathrm{dt}^{2}}=0$ ) at $t=0$, as described in definitions $1-3$, are assumed to be coincident.

Depending on the sign of the second derivative, $\frac{d^{2} \Delta}{d t^{2}}$, on each of the two non-resonant sections, which are adjacent to this resonance $\Delta \approx 0$, three cases of external stability of this resonance can be distinguished.

Let us consider the evolution of $\Delta(t)$ in the positive nonresonant range of values $\Delta>0$ at an externally stable resonance. If the condition $\frac{d \Delta}{d t}<0$ is met, it is possible here to distinguish three stable cases of the system behavior (1)-(2): Case 1: $\frac{d^{2} \Delta}{d t^{2}}>0$ is the case of weak nonlinear external stability (Fig. 1, curve 1).

Case 2: $\frac{d^{2} \Delta}{d t^{2}}=0$ is the case of linear external stability (Fig. 1 , line 2).

Case 3: $\frac{d^{2} \Delta}{d t^{2}}<0$ is the case of strong nonlinear external stability (Fig. 1, curve 3).

Let us consider the evolution of $\Delta(t)$ in the negative nonresonant range of values $\Delta$ at an externally stable resonance. If the condition $\frac{d \Delta}{d t}>0$ is met, it is also possible here to distinguish three stable cases of the system behavior (1)-(2): 
Case 4: $\frac{d^{2} \Delta}{d t^{2}}<0$ is the case of weak nonlinear external stability (Fig. 2, curve 1).

Case 5: $\frac{d^{2} \Delta}{d t^{2}}=0$ is the case of linear external stability (Fig. 2, line 2).

Case 3: $\frac{d^{2} \Delta}{d t^{2}}>0$ is the case of strong nonlinear external stability (Fig. 2, curve 3).

$\Delta(t), s^{-1}$

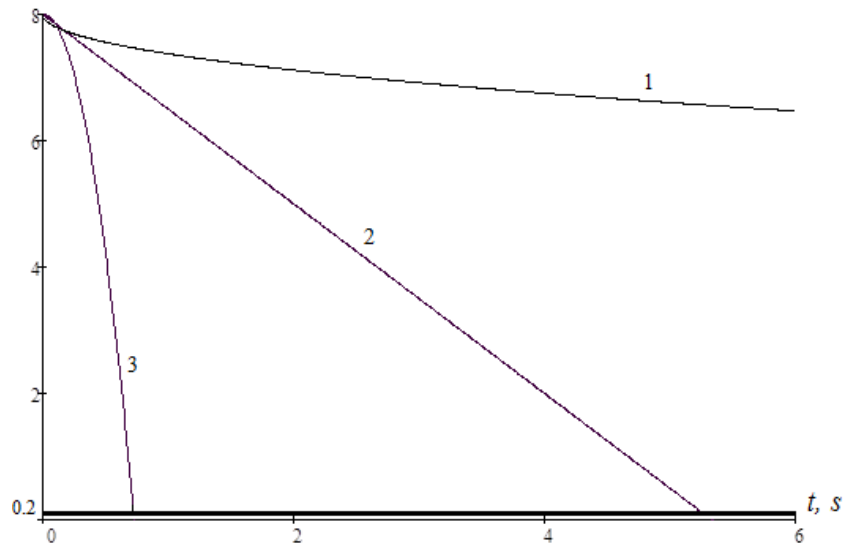

Fig. 1. Change $\Delta(t)>0$ at an externally stable resonance

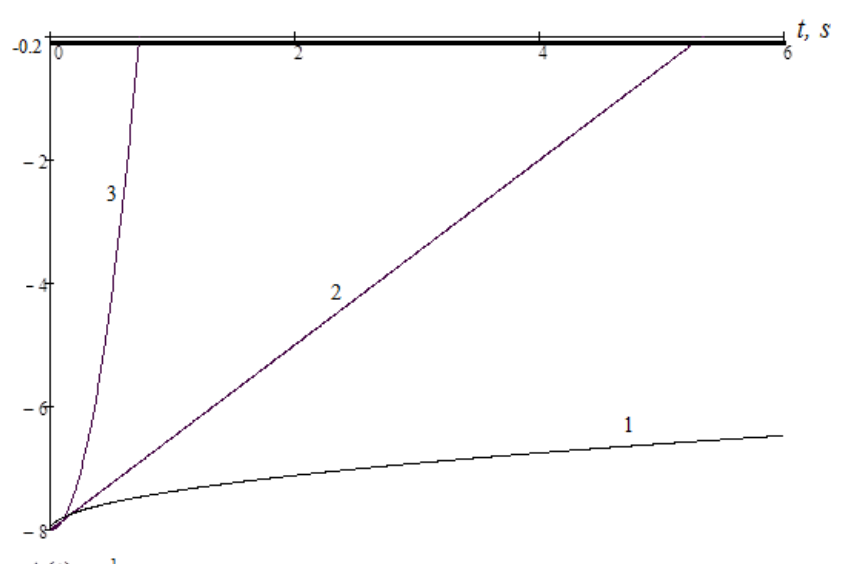

$\Delta(t), s^{-1}$

Fig. 2. Change $\Delta(t)<0$ at an externally stable resonance

\subsection{Strong, weak, and linear external resonance instability}

Similarly, let us introduce some concepts.

Definition 4. The linear monotonic external instability of an individual resonance $\Delta \approx 0$ is understood as a monotonous convergence $\Delta$ from a small resonant area that occurs in a given non-resonant area, which is adjacent to the resonance by means of linear dependence $\Delta(t)$.

Definition 5. The weak nonlinear or simple-weak monotonic external instability of an individual resonance $\Delta \approx 0$ is understood as a monotonous increase $|\Delta|$, occurring in a given non-resonant area that is adjacent to the resonance, which ensures the achievement of a specific value $|\Delta(\mathrm{t})|>|\Delta(0)|$ for a longer time interval than with linear external instability.

Definition 6. The strong nonlinear or simple-strong monotonic external instability of an individual resonance $\Delta \approx 0$ is understood as a monotonous increase $|\Delta|$ occurring in a given non-resonant area that is adjacent to the resonance, which ensures the achievement of a specific value $|\Delta(\mathrm{t})|>|\Delta(0)|$ for a longer time interval than with linear external instability.

Similarly, depending on the sign of the second derivative $\frac{d^{2} \Delta}{d t^{2}}$ on each of the two non-resonant sections, which are adjacent to this resonance $\Delta \approx 0$, three cases of external instability of the resonance under consideration can be distinguished.

Let us consider the evolution of $\Delta(t)$ in the positive nonresonant range of values $\Delta$ at an externally instable resonance. If the condition $\frac{d \Delta}{d t}>0$ is met, it is possible here to distinguish three instable cases of the system behavior (1)-(2):

Case 7: $\frac{d^{2} \Delta}{d t^{2}}<0$ is the case of weak nonlinear external instability (Fig. 3, curve 1).

Case 8: $\frac{d^{2} \Delta}{d t^{2}}=0$ is the case of linear external instability (Fig. 3, line 2).

Case 9: $\frac{d^{2} \Delta}{d t^{2}}>0$ is the case of strong nonlinear external instability (Fig. 3, curve 3).

Further, let us consider the evolution of $\Delta(t)$ in the negative non-resonant range of values, $\Delta$, at an externally instable resonance. If the condition $\frac{d \Delta}{d t}<0$ is met, it is also possible here to distinguish three instable cases of the system behavior (1)-(2):

Case 10: $\frac{d^{2} \Delta}{d t^{2}}>0$ is the case of weak nonlinear external instability (Fig. 4, curve 1).

Case 11: $\frac{d^{2} \Delta}{d t^{2}}=0$ is the case of linear external instability (Fig. 4, line 2). 


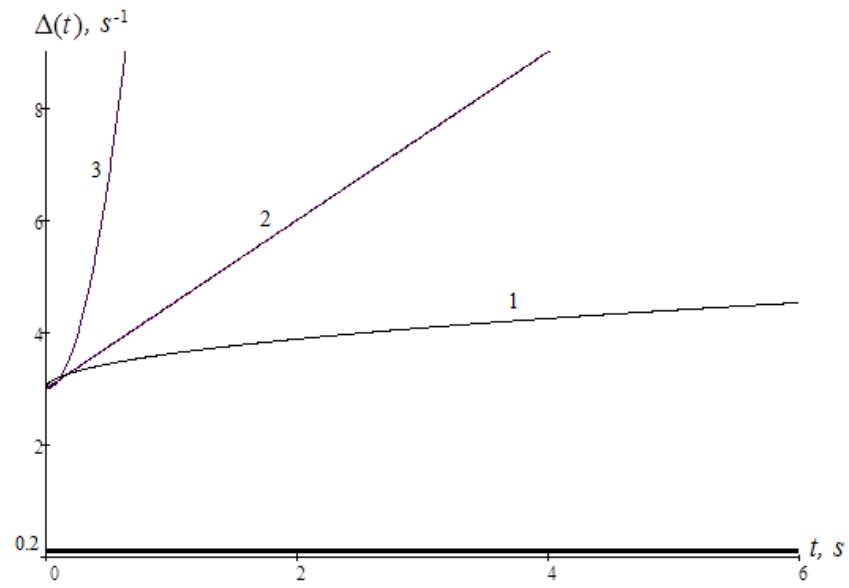

Fig. 3. Change $\Delta(t)>0$ at an externally unstable resonance

Case 12: $\frac{d^{2} \Delta}{d t^{2}}<0$ is the case of strong nonlinear external instability (Fig. 4, curve 3).

Fig.1-4 show the thick horizontal lines limiting the values of small resonant values $\Delta$, which have an order $\Delta=o(\sqrt{\varepsilon})$ in the general case. Fig. 1-4 assume that $\sqrt{\varepsilon}=0.2$.

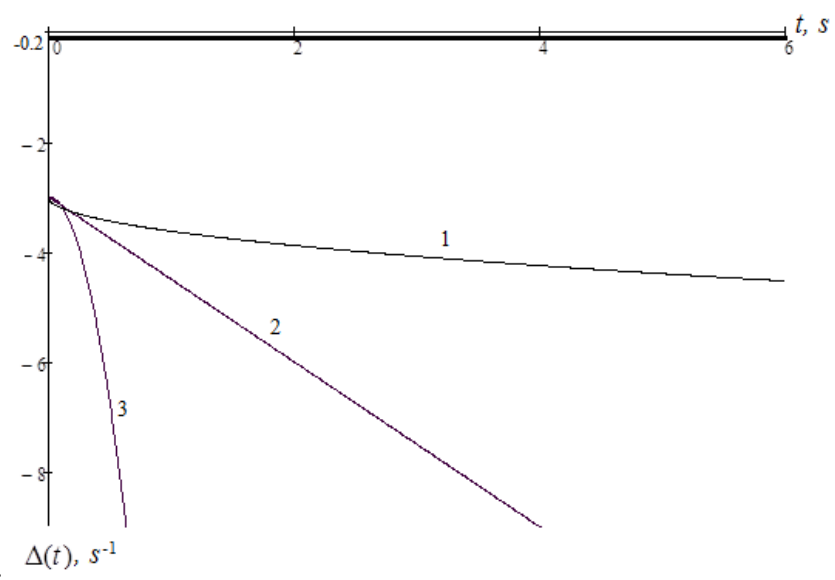

Fig. 4. Change $\Delta(t)<0$ at an externally unstable resonance

\section{Conditions of Monotonic External Stability and Instability of the Resonance}

\subsection{Conditions of monotonic external stability of the resonance}

First, let us consider the conditions of strong external stability of the resonance $\Delta(\omega) \approx 0$.

The following theorem holds.

Theorem 1 (a necessary condition for strong monotonic external stability of the resonance). In order for the resonance $\Delta(\omega) \approx 0$ in the system (1)-(2) to be a strong externally stable one, the following condition should be met:

$$
\frac{d \Delta}{d t} \cdot \frac{d^{2} \Delta}{d t^{2}}>0
$$

Proof of necessity:

It is necessary to consider separately the cases of changes $\Delta(t)$ at $\Delta(t)>0$ and at $\Delta(t)<0$.

The satisfaction of equalities $\frac{d \Delta}{d t}<0$ and $\frac{d^{2} \Delta}{d t^{2}}<0$ is observed on the non-resonant interval, $t \in\left[t_{\mathrm{o}}, t_{\mathrm{r}}\right)$, when the positive values $\Delta(t)$ change with strong external stability of the resonance. Hence, the condition $\frac{d \Delta}{d t} \cdot \frac{d^{2} \Delta}{d t^{2}}>0$ is valid.

The satisfaction of equalities $\frac{d \Delta}{d t}>0$ and $\frac{d^{2} \Delta}{d t^{2}}>0$ is observed on the non-resonant interval, $t \in\left[t_{\mathrm{o}}, t_{\mathrm{r}}\right)$, when the negative values $\Delta(t)$ change with strong external stability of the resonance. Hence, in this case, the condition $\frac{d \Delta}{d t} \cdot \frac{d^{2} \Delta}{d t^{2}}>0$ is also satisfied. The theorem is proved.

Thus, condition (3) is necessary for strong external stability of the resonance when $\Delta(t)$ changes on two non-resonant intervals, $t \in\left[t_{\mathrm{o}}, t_{\mathrm{r}}\right)$, which are adjacent to a given resonance $\Delta \approx 0$. Therefore, condition (3) is a property that characterizes the strong external stability of a given resonance.

Let us note that the converse statement of this theorem is not true in the general case. Genuinely, for example, if condition (3) is satisfied, both the stable case $\left(\frac{d \Delta}{d t}<0, \frac{d^{2} \Delta}{d t^{2}}<0\right)$ and the unstable case $\left(\frac{d \Delta}{d t}>0, \frac{d^{2} \Delta}{d t^{2}}>0\right)$ can be observed at $\Delta(t)>0$.

Let us define the necessary and sufficient conditions for the strong external stability of this resonance.

To this end, let us introduce the following diagonal derivative matrix:

$$
D=\left(\begin{array}{cc}
\frac{d \Delta}{d t} & 0 \\
0 & \frac{d^{2} \Delta}{d t^{2}}
\end{array}\right) .
$$

The following theorem holds.

Theorem 2 (a necessary and sufficient condition for strong monotonic external stability of the resonance). In order for the resonance $\Delta(\omega) \approx 0$ in the system (1)-(2) to be a strong externally stable resonance, it is necessary and sufficient that the principal diagonal minors of the diagonal matrix (4) have the following signs:

$\mathrm{i}$ if $\Delta(t)>0$, then the minors $\frac{d \Delta}{d t}<0, \frac{d \Delta}{d t} \cdot \frac{d^{2} \Delta}{d t^{2}}>0$, 
ii if $\Delta(t)<0$, then the minors $\frac{d \Delta}{d t}>0, \frac{d \Delta}{d t} \cdot \frac{d^{2} \Delta}{d t^{2}}>0$.

\section{Proof of necessity:}

It is necessary to consider separately the cases of changes $\Delta(t)$ at $\Delta(t)>0$ and at $\Delta(t)<0$.

The satisfaction of equalities $\frac{d \Delta}{d t}<0$ and $\frac{d^{2} \Delta}{d t^{2}}<0$ is observed on the non-resonant interval, $t \in\left[t_{0}, t_{\mathrm{r}}\right)$, when the positive values $\Delta(t)$, change with strong external stability of the resonance. Hence, the principal diagonal minors of the diagonal matrix (4) at $\Delta(t)>0$ have signs $\frac{d \Delta}{d t}<0$, $\frac{d \Delta}{d t} \cdot \frac{d^{2} \Delta}{d t^{2}}>0$

The satisfaction of equalities $\frac{d \Delta}{d t}>0$ and $\frac{d^{2} \Delta}{d t^{2}}>0$ is observed on the non-resonant interval, $t \in\left[t_{0}, t_{\mathrm{r}}\right)$, when the negative values $\Delta(t)$, change with strong external stability of the resonance. Hence, the principal diagonal minors of the diagonal matrix (4) at $\Delta(t)<0$ have signs $\frac{d \Delta}{d t}>0$, $\frac{d \Delta}{d t} \cdot \frac{d^{2} \Delta}{d t^{2}}>0$.

The necessity of the theorem is proved.

Proof of sufficiency:

It is necessary to consider separately the cases of changes $\Delta(t)$ at $\Delta(t)>0$ and at $\Delta(t)<0$.

The minors of the diagonal matrix (4) will have signs $\frac{d \Delta}{d t}<0, \frac{d \Delta}{d t} \cdot \frac{d^{2} \Delta}{d t^{2}}>0$ on the non-resonant interval $t \in\left[t_{0}, t_{\mathrm{r}}\right)$, when the positive values of $\Delta(t)$ change. Therefore, on this interval, the first and second derivatives will have the signs $\frac{d \Delta}{d t}<0, \frac{d^{2} \Delta}{d t^{2}}<0$. In this case, the resonance $\Delta \approx 0$ is a strong externally stable resonance. Genuinely, when the conditions $\Delta(t)>0, \frac{d \Delta}{d t}<0$, and $\frac{d^{2} \Delta}{d t^{2}}<0$ are met, the monotonous convergence $\Delta$ to a small resonant area occurring in a given, adjacent to the resonance non-resonant area $\Delta(t)>0$, occurs for a shorter time interval than with linear external stability, when the conditions $\Delta(t)>0, \frac{d \Delta}{d t}<0$, and $\frac{d^{2} \Delta}{d t^{2}}=0$ are met.

The minors of the diagonal matrix (4) will have signs $\frac{d \Delta}{d t}>0, \frac{d \Delta}{d t} \cdot \frac{d^{2} \Delta}{d t^{2}}>0$ on the non-resonant interval $t \in\left[t_{0}, t_{\mathrm{r}}\right)$, when the positive values of $\Delta(t)$ change. Therefore, on this interval, the first and second derivatives will have the signs $\frac{d \Delta}{d t}>0$ and $\frac{d^{2} \Delta}{d t^{2}}>0$. In this case, the resonance $\Delta \approx 0$ is also a strong externally stable resonance. Genuinely, when the conditions $\Delta(t)<0, \frac{d \Delta}{d t}>0$, and $\frac{d^{2} \Delta}{d t^{2}}>0$ are met the monotonous convergence $\Delta$ to a small resonant area occurring in a given, adjacent to the resonance non-resonant area $\Delta(t)<0$, occurs for a shorter time interval than with linear external stability, when the conditions $\Delta(t)<0, \frac{d \Delta}{d t}>0$, and $\frac{d^{2} \Delta}{d t^{2}}=0$ are met.

The sufficiency of the theorem is proved.

Second, let us consider the conditions of weak external stability of the resonance, $\Delta(\omega) \approx 0$.

The following theorem also holds.

Theorem 3 (a necessary condition for weak monotonic external stability of the resonance). In order for the resonance $\Delta(\omega) \approx 0$ in the system (1)-(2) to be a weak externally stable one, the following condition should be met:

$$
\frac{d \Delta}{d t} \cdot \frac{d^{2} \Delta}{d t^{2}}<0 .
$$

\section{Proof of necessity:}

It is necessary to consider separately the cases of changes $\Delta(t)$ at $\Delta(t)>0$ and at $\Delta(t)<0$.

The satisfaction of equalities $\frac{d \Delta}{d t}<0$ and $\frac{d^{2} \Delta}{d t^{2}}>0$ is observed on the non-resonant interval, $t \in\left[t_{0}, t_{\mathrm{r}}\right)$, when the positive values $\Delta(t)$, change with weak external stability of the resonance. Hence, the condition $\frac{d \Delta}{d t} \cdot \frac{d^{2} \Delta}{d t^{2}}<0$ is valid.

The satisfaction of equalities $\frac{d \Delta}{d t}>0$ and $\frac{d^{2} \Delta}{d t^{2}}<0$ is observed on the non-resonant interval, $t \in\left[t_{\mathrm{o}}, t_{\mathrm{r}}\right)$, when the negative values $\Delta(t)$, change with strong external stability of the resonance. Hence, in this case, the condition $\frac{d \Delta}{d t} \cdot \frac{d^{2} \Delta}{d t^{2}}>0$ is also satisfied. The theorem is proved.

Thus, condition (4) is necessary for strong external stability of the resonance when $\Delta(t)$ changes on two non-resonant intervals $t \in\left[t_{0}, t_{\mathrm{r}}\right)$, which are adjacent to a given resonance $\Delta \approx 0$. Therefore, condition (4) is a property that characterizes the weak external stability of a given resonance in the system 
(1)-(2).

Let us note that the converse statement of theorem 2 is not true in the general case. Genuinely, for example, if condition (4) is satisfied, both the stable case $\left(\frac{d \Delta}{d t}<0, \frac{d^{2} \Delta}{d t^{2}}>0\right)$ and the unstable case $\left(\frac{d \Delta}{d t}>0, \frac{d^{2} \Delta}{d t^{2}}<0\right)$ can be observed at $\Delta(t)>0$.

Let us define the necessary and sufficient conditions for the weak external stability of this resonance.

The following theorem also holds.

Theorem 4 (a necessary and sufficient condition for weak monotonic external stability of the resonance).

In order for the resonance $\Delta(\omega) \approx 0$ in the system (1)-(2) to be a weak externally stable resonance, it is necessary and sufficient that the principal diagonal minors of the diagonal matrix (4) have the following signs:

$\mathrm{i}$ if $\Delta(t)>0$, then the minors $\frac{d \Delta}{d t}<0, \frac{d \Delta}{d t} \cdot \frac{d^{2} \Delta}{d t^{2}}<0$,

ii if $\Delta(t)<0$, then the minors $\frac{d \Delta}{d t}>0, \frac{d \Delta}{d t} \cdot \frac{d^{2} \Delta}{d t^{2}}<0$.

\section{Proof of necessity:}

It is necessary to consider separately the cases of changes $\Delta(t)$ at $\Delta(t)>0$ and at $\Delta(t)<0$.

The satisfaction of equalities $\frac{d \Delta}{d t}<0$ and $\frac{d^{2} \Delta}{d t^{2}}>0$ is observed on the non-resonant interval $t \in\left[t_{0}, t_{\mathrm{r}}\right)$, when the positive values $\Delta(t)$, change with weak external stability of the resonance. Hence, the principal diagonal minors of the diagonal matrix (4) at $\Delta(t)>0$ have signs $\frac{d \Delta}{d t}<0$, $\frac{d \Delta}{d t} \cdot \frac{d^{2} \Delta}{d t^{2}}<0$

The satisfaction of equalities $\frac{d \Delta}{d t}>0$ and $\frac{d^{2} \Delta}{d t^{2}}<0$ is observed on the non-resonant interval, $t \in\left[t_{\mathrm{o}}, t_{\mathrm{r}}\right)$, when the negative values $\Delta(t)$, change with strong external stability of the resonance. Hence, the principal diagonal minors of the diagonal matrix (4) at $\Delta(t)<0$ have signs $\frac{d \Delta}{d t}>0$, $\frac{d \Delta}{d t} \cdot \frac{d^{2} \Delta}{d t^{2}}<0$

The necessity of the theorem is proved.

Proof of sufficiency:

It is necessary to consider separately the cases of changes $\Delta(t)$ at $\Delta(t)>0$ and at $\Delta(t)<0$.

The minors of the diagonal matrix (4) will have signs $\frac{d \Delta}{d t}<0, \frac{d \Delta}{d t} \cdot \frac{d^{2} \Delta}{d t^{2}}<0$ on the non-resonant interval $t \in\left[t_{\mathrm{o}}, t_{\mathrm{r}}\right)$, when the positive values of $\Delta(t)$ change. Therefore, on this interval, the first and second derivatives will have the signs $\frac{d \Delta}{d t}<0, \frac{d^{2} \Delta}{d t^{2}}>0$. In this case, the resonance, $\Delta \approx 0$, is a weak externally stable resonance. Genuinely, when the conditions $\Delta(t)>0, \frac{d \Delta}{d t}<0$, and $\frac{d^{2} \Delta}{d t^{2}}>0$ are met, the monotonous convergence $\Delta$ to a small resonant area occurring in a given, adjacent to the resonance non-resonant area $\Delta(t)>0$ occurs for a longer time interval than with linear external stability, when the conditions $\Delta(t)>0, \frac{d \Delta}{d t}<0$, and $\frac{d^{2} \Delta}{d t^{2}}=0$ are met.

The minors of the diagonal matrix (4) will have signs $\frac{d \Delta}{d t}>0, \frac{d \Delta}{d t} \cdot \frac{d^{2} \Delta}{d t^{2}}<0$ on the non-resonant interval $t \in\left[t_{\mathrm{o}}, t_{\mathrm{r}}\right)$, when the positive values of $\Delta(t)$ change. Therefore, on this interval, the first and second derivatives will have the signs $\frac{d \Delta}{d t}>0$ and $\frac{d^{2} \Delta}{d t^{2}}<0$. In this case, the resonance $\Delta \approx 0$ is also a weak externally stable resonance. Genuinely, when the conditions $\Delta(t)<0, \frac{d \Delta}{d t}>0$, and $\frac{d^{2} \Delta}{d t^{2}}<0$, are met, the monotonous convergence $\Delta$ to a small resonant area occurring in a given, adjacent to the resonance, non-resonant area $\Delta(t)<0$ occurs for a longer time interval than with linear external stability, when the conditions $\Delta(t)<0, \frac{d \Delta}{d t}>0$, and $\frac{d^{2} \Delta}{d t^{2}}=0$ are met.

The sufficiency of the theorem is proved.

\subsection{Conditions of monotonic external instability of the resonance}

Similarly to the stability analysis, let us first consider the conditions of strong instability of the resonance $\Delta(\omega) \approx 0$.

The following theorem on strong external instability holds. Theorem 5 (a necessary condition for strong monotonic external instability of the resonance). In order for the resonance $\Delta(\omega) \approx 0$ in the system (1)-(2) to be a strong externally instable one, the following condition should be met:

$$
\frac{d \Delta}{d t} \cdot \frac{d^{2} \Delta}{d t^{2}}>0
$$

\section{Proof of necessity:}


Let us consider separately the cases of changes $\Delta(t)$ at $\Delta(t)>0$ and at $\Delta(t)<0$.

The simultaneous satisfaction of equalities $\frac{d \Delta}{d t}>0$ and $\frac{d^{2} \Delta}{d t^{2}}>0$ is observed on the non-resonant segment $t \in\left[t_{\mathrm{o}}, t_{\mathrm{k}}\right]$, where $t_{\mathrm{k}}$ is the final time value for this interval, when the positive values $\Delta(t)$ change with strong external instability of the resonance. Hence, the condition $\frac{d \Delta}{d t} \cdot \frac{d^{2} \Delta}{d t^{2}}>0$ is valid.

The simultaneous satisfaction of equalities $\frac{d \Delta}{d t}<0$ and $\frac{d^{2} \Delta}{d t^{2}}<0$ is observed on the non-resonant segment, $t \in\left[t_{\mathrm{o}}, t_{\mathrm{k}}\right]$, when the negative values $\Delta(t)$ change with strong external stability of the resonance. Hence, in this case, the condition $\frac{d \Delta}{d t} \cdot \frac{d^{2} \Delta}{d t^{2}}>0$ is also satisfied. The theorem is proved.

Here, it should also be noted that the converse statement of this theorem is not true in the general case. Genuinely, for example, if condition (10) is satisfied, both the stable case $\left(\frac{d \Delta}{d t}<0, \frac{d^{2} \Delta}{d t^{2}}<0\right)$ and the unstable case $\left(\frac{d \Delta}{d t}>0\right.$, $\left.\frac{d^{2} \Delta}{d t^{2}}>0\right)$ can be observed at $\Delta(t)>0$.

Let us define the necessary and sufficient conditions for the strong external instability of this resonance.

The following theorem holds.

Theorem 6 (a necessary and sufficient condition for strong monotonic external instability of the resonance). In order for the resonance $\Delta(\omega) \approx 0$ in the system (1)-(2) to be a strong externally instable resonance, it is necessary and sufficient that the principal diagonal minors of the diagonal matrix (4) have the following signs:

$\mathrm{i}$ if $\Delta(t)>0$, then the minors $\frac{d \Delta}{d t}>0, \frac{d \Delta}{d t} \cdot \frac{d^{2} \Delta}{d t^{2}}>0$,

ii if $\Delta(t)<0$, then the minors $\frac{d \Delta}{d t}<0, \frac{d \Delta}{d t} \cdot \frac{d^{2} \Delta}{d t^{2}}>0$.

\section{Proof of necessity:}

It is necessary to consider separately the cases of changes $\Delta(t)$ at $\Delta(t)>0$ and at $\Delta(t)<0$

The satisfaction of equalities $\frac{d \Delta}{d t}>0$ and $\frac{d^{2} \Delta}{d t^{2}}>0$ is observed on the non-resonant interval $t \in\left[t_{0}, t_{\mathrm{k}}\right]$, when the positive values $\Delta(t)$ change with strong external instability of the resonance. Hence, the principal diagonal minors of the diagonal matrix (4) at $\Delta(t)>0$ have signs $\frac{d \Delta}{d t}>0$, $\frac{d \Delta}{d t} \cdot \frac{d^{2} \Delta}{d t^{2}}>0$

The satisfaction of equalities $\frac{d \Delta}{d t}<0$ and $\frac{d^{2} \Delta}{d t^{2}}<0$ is observed on the non-resonant interval $t \in\left[t_{\mathrm{o}}, t_{\mathrm{k}}\right]$, when the negative values $\Delta(t)$ change with strong external stability of the resonance. Hence, the principal diagonal minors of the diagonal matrix (4) at $\Delta(t)<0$ have signs $\frac{d \Delta}{d t}<0$, $\frac{d \Delta}{d t} \cdot \frac{d^{2} \Delta}{d t^{2}}>0$

The necessity of the theorem is proved.

Proof of sufficiency:

It is necessary to consider separately the cases of changes $\Delta(t)$ at $\Delta(t)>0$ and at $\Delta(t)<0$.

The minors of the diagonal matrix (4) will have signs $\frac{d \Delta}{d t}>0, \frac{d \Delta}{d t} \cdot \frac{d^{2} \Delta}{d t^{2}}>0$ on the non-resonant interval $t \in\left[t_{\mathrm{o}}, t_{\mathrm{k}}\right]$, when the positive values of $\Delta(t)$ change. Therefore, on this interval, the first and second derivatives will have the signs $\frac{d \Delta}{d t}>0, \frac{d^{2} \Delta}{d t^{2}}>0$. In this case, the resonance, $\Delta \approx 0$, is a strong externally instable resonance. Genuinely, when the conditions $\Delta(t)>0, \frac{d \Delta}{d t}>0$, and $\frac{d^{2} \Delta}{d t^{2}}>0$, are met, the monotonic increase $|\Delta|$ occurs in a given, adjacent to the resonance non-resonant area $\Delta(t)>0$, and ensures the achievement of a specific value $|\Delta(\mathrm{t})|>|\Delta(0)|$ for a shorter time interval than with the linear external instability observed when the conditions $\Delta(t)>0, \frac{d \Delta}{d t}>0$, and $\frac{d^{2} \Delta}{d t^{2}}=0$ are met.

The minors of the diagonal matrix (4) will have signs $\frac{d \Delta}{d t}<0, \frac{d \Delta}{d t} \cdot \frac{d^{2} \Delta}{d t^{2}}>0$ on the non-resonant interval $t \in\left[t_{\mathrm{o}}, t_{\mathrm{k}}\right]$, when the negative values of $\Delta(t)$ change. Therefore, on this interval, the first and second derivatives will have the signs $\frac{d \Delta}{d t}<0$ and $\frac{d^{2} \Delta}{d t^{2}}<0$. In this case, the resonance $\Delta \approx 0$ is also a strong externally instable resonance. Genuinely, when the conditions $\Delta(t)<0, \frac{d \Delta}{d t}<0$, and $\frac{d^{2} \Delta}{d t^{2}}<0$, are met, the monotonic increase $|\Delta|$ occurs in a given, adjacent to the 
resonance non-resonant area $\Delta(t)<0$, and ensures the achievement of a specific value $|\Delta(\mathrm{t})|>|\Delta(0)|$ for a shorter time interval than with the linear external instability observed when the conditions $\Delta(t)<0, \frac{d \Delta}{d t}<0$, and $\frac{d^{2} \Delta}{d t^{2}}=0$ are met.

The theorem is proved.

Further, let us define the conditions for weak external instability of the resonance $\Delta(\omega) \approx 0$.

The theorem holds.

Theorem 7 (a necessary condition for weak monotonic external instability of the resonance). In order for the resonance $\Delta(\omega) \approx 0$ in the system (1)-(2) to be a weak externally instable one, the following condition should be met:

$$
\frac{d \Delta}{d t} \cdot \frac{d^{2} \Delta}{d t^{2}}<0
$$

\section{Proof of necessity:}

Let us consider separately the cases of changes $\Delta(t)$ at $\Delta(t)>0$ and at $\Delta(t)<0$.

The simultaneous satisfaction of equalities $\frac{d \Delta}{d t}>0$ and $\frac{\mathrm{d}^{2} \Delta}{\mathrm{dt}^{2}}<0$ is observed on the non-resonant segment $t \in\left[t_{0}, t_{\mathrm{k}}\right]$, where $t_{\mathrm{k}}$ is the final time value for this interval, when the positive values $\Delta(t)$ change with weak external instability of the resonance. Hence, the condition $\frac{d \Delta}{d t} \cdot \frac{d^{2} \Delta}{d t^{2}}<0$ is valid.

The simultaneous satisfaction of equalities $\frac{d \Delta}{d t}<0$ and $\frac{\mathrm{d}^{2} \Delta}{\mathrm{dt}^{2}}>0$ is observed on the non-resonant segment $t \in\left[t_{\mathrm{o}}, t_{\mathrm{k}}\right]$, when the negative values $\Delta(t)$ change with weak external stability of the resonance. Hence, in this case, the condition $\frac{d \Delta}{d t} \cdot \frac{d^{2} \Delta}{d t^{2}}<0$ is also satisfied. The theorem is proved.

The converse statement of this theorem is not true in the general case. Genuinely, for example, if condition (6) is satisfied, both the stable case $\left(\frac{d \Delta}{d t}<0, \frac{\mathrm{d}^{2} \Delta}{\mathrm{dt}^{2}}>0\right)$ and the unstable case $\left(\frac{d \Delta}{d t}>0, \frac{\mathrm{d}^{2} \Delta}{\mathrm{dt}^{2}}<0\right)$ can be observed at $\Delta(t)>0$.

Let us define the necessary and sufficient conditions for the weak external instability of this resonance.

The following theorem holds.

Theorem 8 (a necessary and sufficient condition for weak monotonic external instability of the resonance). In order for the resonance $\Delta(\omega) \approx 0$ in the system (1)-(2) to be a weak externally instable resonance, it is necessary and sufficient that the principal diagonal minors of the diagonal matrix (4) have the following signs:

i if $\Delta(t)>0$, then the minors $\frac{d \Delta}{d t}>0, \frac{d \Delta}{d t} \cdot \frac{d^{2} \Delta}{d t^{2}}<0$,

ii if $\Delta(t)<0$, then the minors $\frac{d \Delta}{d t}<0, \frac{d \Delta}{d t} \cdot \frac{d^{2} \Delta}{d t^{2}}<0$.

Proof of necessity:

It is necessary to consider separately the cases of changes $\Delta(t)$ at $\Delta(t)>0$ and at $\Delta(t)<0$.

The satisfaction of equalities $\frac{d \Delta}{d t}>0$ and $\frac{d^{2} \Delta}{d t^{2}}<0$ is observed on the non-resonant interval $t \in\left[t_{0}, t_{\mathrm{k}}\right]$, when the positive values $\Delta(t)$ change with weak external instability of the resonance. Hence, the principal diagonal minors of the diagonal matrix (4) at $\Delta(t)>0$ have signs $\frac{d \Delta}{d t}>0$, $\frac{d \Delta}{d t} \cdot \frac{d^{2} \Delta}{d t^{2}}<0$

The satisfaction of equalities $\frac{d \Delta}{d t}<0$ and $\frac{d^{2} \Delta}{d t^{2}}>0$ is observed on the non-resonant interval $t \in\left[t_{\mathrm{o}}, t_{\mathrm{k}}\right]$, when the negative values $\Delta(t)$ change with weak external stability of the resonance. Hence, the principal diagonal minors of the diagonal matrix (4) at $\Delta(t)<0$ have signs $\frac{d \Delta}{d t}<0$, $\frac{d \Delta}{d t} \cdot \frac{d^{2} \Delta}{d t^{2}}<0$

The necessity of the theorem is proved.

Proof of sufficiency:

It is necessary to consider separately the cases of changes $\Delta(t)$ at $\Delta(t)>0$ and at $\Delta(t)<0$.

The principal diagonal minors of the matrix (4) will have signs $\frac{d \Delta}{d t}>0, \frac{d \Delta}{d t} \cdot \frac{d^{2} \Delta}{d t^{2}}<0$ on the non-resonant interval $t \in\left[t_{\mathrm{o}}, t_{\mathrm{k}}\right]$, when the positive values of $\Delta(t)$ change. Therefore, on this interval, the first and second derivatives will have the signs $\frac{d \Delta}{d t}>0, \frac{d^{2} \Delta}{d t^{2}}<0$. In this case, the resonance $\Delta \approx 0$ is a weak externally instable resonance. Genuinely, when the conditions $\Delta(t)>0, \frac{d \Delta}{d t}>0$, and $\frac{d^{2} \Delta}{d t^{2}}<0$ are met, the monotonic increase $|\Delta|$ occurs in a given, adjacent to the resonance non-resonant area $\Delta(t)>0$, and ensures the achievement of a specific value $|\Delta(\mathrm{t})|>|\Delta(0)|$ for a longer time interval than with the linear external instability observed 
when the conditions $\Delta(t)>0, \frac{d \Delta}{d t}>0$, and $\frac{d^{2} \Delta}{d t^{2}}=0$ are met. The main diagonal minors of the matrix (4) will have signs $\frac{d \Delta}{d t}<0, \frac{d \Delta}{d t} \cdot \frac{d^{2} \Delta}{d t^{2}}<0$ on the non-resonant interval $t \in\left[t_{0}, t_{\mathrm{k}}\right]$, when the positive values of $\Delta(t)$ change. Therefore, on this interval, the first and second derivatives will have the signs $\frac{d \Delta}{d t}<0$ and $\frac{d^{2} \Delta}{d t^{2}}>0$. In this case, the resonance $\Delta \approx 0$ is also a weak externally instable resonance. Genuinely, when the conditions $\Delta(t)<0, \frac{d \Delta}{d t}<0$, and $\frac{d^{2} \Delta}{d t^{2}}>0$ are met, the monotonic increase $|\Delta|$ occurs in a given, adjacent to the resonance non-resonant area $\Delta(t)<0$, and ensures the achievement of a specific value $|\Delta(\mathrm{t})|>|\Delta(0)|$, for a longer time interval than with the linear external instability observed when the conditions $\Delta(t)<0, \frac{d \Delta}{d t}<0$, and $\frac{d^{2} \Delta}{d t^{2}}=0$ are met. The theorem is proved.

\section{Analysis of Monotonic External Stability in the Motion of an Asymmetric Lagrange Top}

Let us consider the analysis of the weak and strong monotonic stability of the resonance on the example of the problem of the motion of an asymmetric rigid body (RB) relative to a fixed point. Let a heavy rigid body with small mass asymmetry move relative to a fixed point in the vicinity of a statically stable equilibrium position. It is assumed that a $\mathrm{RB}$ is affected by a considerable nonlinear restoring moment and a small perturbing moment, the vector of which is motionless in the frame references, associated this RB. As a result of the approximation of the restoring moment by a polynomial of the third degree in the nutation angle and the subsequent averaging of the system of equations of motion of the rigid body, it is possible to obtain a complete picture of the evolutionary motion of the RB. The decisive influence on the motion of the RB is exerted by a lower-order resonance, called the principal resonance.

The evolutionary resonance effects in the rotation of the heavy rigid body with small asymmetry relative to a fixed point in this problem in the case of small nutation angles were considered in the paper [17]. However, this work did not investigate the monotonic linear, weak and strong external stability of resonances.

After the nutation angle linearization of the equations for the motion of a rigid body, which are averaged over fast variables, a subsystem of equations is obtained:

$$
\begin{gathered}
\frac{d \omega_{z}}{d t}=\varepsilon^{2} \frac{G \Delta y M_{p} \cos \Phi_{0}}{2 I_{z} I \omega_{\theta} \Delta}, \\
\frac{d \gamma}{d t}=\Delta .
\end{gathered}
$$

where $\varepsilon$ is a small parameter; $\omega_{z}>0$ is the angular velocity of the RB relative to the main central coupled longitudinal axis; $\gamma$ is a fast variable; $G$ is the RB gravity; $\Delta y$ is a small displacement of the center of mass relative to the $z$-axis; $M_{p}$ is a small perturbing moment; $M_{p}=\sqrt{\left(\Delta y G+\Delta M_{x}\right)^{2}+\left(\Delta M_{y}\right)^{2}}, \quad \Delta M_{x}, \Delta M_{y}$ are small sign-constant moments relative to the main central coupled $x$ and $y$-axes, respectively; $\cos \Phi_{0}=-\Delta M_{y} / M_{p}, I_{z}$, and $I=I_{x}=I_{y}$ are the main axial moments of inertia of the RB relative to the corresponding axes; $\omega_{1}=0.5 I_{z} I^{-1} \omega_{z}+\omega_{\theta}$, $\omega_{\theta}=\sqrt{0.25 I_{z}^{2} I^{-2} \omega_{z}^{2}+\omega_{0}^{2}}$, and $\omega_{0}$ are the frequency of vibrations of the RB at $\omega_{z}=0$; and $\Delta=\omega_{z}-\omega_{1}$ is the resonant ratio of two frequencies.

When implementing the resonance in the system under consideration (16)-(17), the equality $\Delta=\omega_{z}-\omega_{1}=O(\sqrt{\varepsilon})$ is satisfied.

Let us perform an analysis of the signs of the derivatives $\frac{d \Delta}{d t}$ and $\frac{d^{2} \Delta}{d t^{2}}$ in the system of equations (16)-(17). As a result of differentiating the function $\Delta(t)$ taking into account (16)-(17), the following will be obtained:

$$
\begin{aligned}
\frac{d \Delta}{d t} & =\frac{d \Delta}{d \omega_{z}} \frac{d \omega_{z}}{d t}, \\
\frac{d^{2} \Delta}{d t^{2}} & =\frac{d}{d \omega_{z}}\left(\frac{d \Delta}{d t}\right) \frac{d \omega_{z}}{d t},
\end{aligned}
$$

where $\frac{d \Delta}{d \omega_{z}}=-C_{1} f_{1}\left(\omega_{z}\right), C_{1}=\frac{G \Delta y M_{p} \cos \Phi_{0}}{2 I_{z} I}$,

$f_{1}\left(\omega_{z}\right)=0.25 I_{z} I^{-1} \omega_{z} \omega_{\theta}^{-3} \Delta^{-1}+\omega_{\theta}^{-1} \Delta^{-2}\left(1-0.5 I_{z} I^{-1}-0.25 I_{z}^{2} I^{-2} \omega_{z} \omega_{\theta}^{-1}\right)$,

$\frac{d}{d \omega_{z}}\left(\frac{d \Delta}{d t}\right)=-C_{1} \frac{d}{d \omega_{z}}\left(f_{1}\left(\omega_{z}\right) \frac{d \omega_{z}}{d t}\right)$

Let us analyze the external stability of the resonance in the system (16)-(17). For this purpose, let us use the expressions (18)-(19). A few special cases should be considered.

Case 1. Equation (18) provides that if the conditions $C_{1}<0, \omega_{z}>0$, and $\left(1-0.5 I_{z} I^{-1}-0.25 I_{z}^{2} I^{-2} \omega_{z} \omega_{\theta}^{-1}\right)>0$ are are met simultaneously,

$$
\left|0.25 I_{z} I^{-1} \omega_{z} \omega_{\theta}^{-3} \Delta^{-1}\right|>\left|\omega_{\theta}^{-1} \Delta^{-2}\left(1-0.5 I_{z} I^{-1}-0.25 I_{z}^{2} I^{-2} \omega_{z} \omega_{\theta}^{-1}\right)\right|,
$$

the derivative $\frac{d \Delta}{d \omega_{z}}>0$ on each of the two non-resonant areas $\omega_{z}-\omega_{1}>0$ and $\omega_{z}-\omega_{1}<0$ under consideration. Let the conditions $\frac{d^{2} \Delta}{d t^{2}}>0$ and $\frac{d \Delta}{d \omega_{z}}>0$ be satisfied on the section 
$\omega_{z}-\omega_{1}>0$, according to theorem 6 , we obtain that the resonance $\Delta \approx 0$ is a strong externally instable resonance. If the conditions $\frac{d \Delta}{d \omega_{z}}>0$ and $\frac{d^{2} \Delta}{d t^{2}}>0$ are met simultaneously on the case $\omega_{z}-\omega_{1}<0$, according to theorem 2 , we obtain that the resonance $\Delta \approx 0$ is a strong externally stable resonance.

Case 2. Equation (18) provides that if the conditions $C_{1}>0, \omega_{z}>0$, and $\left(1-0.5 I_{z} I^{-1}-0.25 I_{z}^{2} I^{-2} \omega_{z} \omega_{\theta}^{-1}\right)>0$ are are met simultaneously,

$$
\left|0.25 I_{z} I^{-1} \omega_{z} \omega_{\theta}^{-3} \Delta^{-1}\right|<\left|\omega_{\theta}^{-1} \Delta^{-2}\left(1-0.5 I_{z} I^{-1}-0.25 I_{z}^{2} I^{-2} \omega_{z} \omega_{\theta}^{-1}\right)\right|,
$$

the derivative $\frac{d \Delta}{d \omega_{z}}<0$ in the non-resonant area $\omega_{z}-\omega_{1}>0$; in the area $\omega_{z}-\omega_{1}<0$, the given derivative $\frac{d \Delta}{d \omega_{z}}>0$. Let the conditions $\frac{d \Delta}{d \omega_{z}}<0$ and $\frac{d \Delta}{d \omega_{z}}>0$ be simultaneously satisfied on the case $\omega_{z}-\omega_{1}>0$, according to theorem 4 , we obtain that the resonance $\Delta \approx 0$ is a weak externally stable resonance. If the conditions $\frac{d \Delta}{d \omega_{z}}>0$ and $\frac{d^{2} \Delta}{d t^{2}}<0$ are met simultaneously on the case $\omega_{z}-\omega_{1}<0$, according to theorem 4, we obtain that the resonance $\Delta \approx 0$ is also a strong externally stable resonance.

Case 3. Equation (18) provides that if the conditions $C_{1}<0, \omega_{z}>0$, and $\left(1-0.5 I_{z} I^{-1}-0.25 I_{z}^{2} I^{-2} \omega_{z} \omega_{\theta}^{-1}\right)>0$ are are met simultaneously,

$$
\left|0.25 I_{z} I^{-1} \omega_{z} \omega_{\theta}^{-3} \Delta^{-1}\right|<\left|\omega_{\theta}^{-1} \Delta^{-2}\left(1-0.5 I_{z} I^{-1}-0.25 I_{z}^{2} I^{-2} \omega_{z} \omega_{\theta}^{-1}\right)\right|,
$$

the derivative $\frac{d \Delta}{d \omega_{z}}>0$ in the non-resonant area $\omega_{z}-\omega_{1}>0$; in the area $\omega_{z}-\omega_{1}<0$, the given derivative $\frac{d \Delta}{d \omega_{z}}<0$. Let the conditions $\frac{d \Delta}{d \omega_{z}}>0$ and $\frac{d^{2} \Delta}{d t^{2}}<0$ be simultaneously satisfied on the section $\omega_{z}-\omega_{1}>0$, according to theorem 8 , we obtain that the resonance $\Delta \approx 0$ is a weak externally instable resonance. If the conditions $\frac{d \Delta}{d \omega_{z}}<0$ and $\frac{d^{2} \Delta}{d t^{2}}>0$ are met simultaneously on the case $\omega_{z}-\omega_{1}<0$, according to theorem 8 , we obtain that the resonance $\Delta \approx 0$ is also a strong externally stable resonance.

The analysis of the monotonic external stability of the resonance $\Delta \approx 0$ in the system (16)-(17) shows that the application of the above theorems in the case of positive nonresonant values $\omega_{z}$ allows studying only 16 such frequent cases. By analogy, with negative non-resonant values of $\omega_{z}$, let us also obtain the other 16 special cases.
Thus, the application of the conditions presented here makes it possible to analyze the monotonic external linear, weak and strong stability of the principal resonance under the perturbed motion of the heavy rigid body relative to a fixed point. At the same time, the mechanical reason for the realization of these types of external stability of resonance is the effect of perturbations on the RB. These are disturbances caused by a small moment with a constant modulus, as well as a small moment from the displacement of the center of mass relative to the longitudinal axis of the rigid body. Note that, in contrast to work [17], in this problem, after non-resonant averaging on the right-hand side of the equation (16), the second approximation should be a continuous differentiable function of constant sign.

\section{Discussion}

It is known [12-14] that when obtaining ordinary differential equations for slow variables, in the second approximation with non-resonant averaging, terms with a resonant frequency ratio in the denominator are obtained. Suppose the first approximation of the averaging method in the equation for slow variables is equal to zero, and the evolution of these variables is determined by the terms of the second approximation.

The paper deals with a perturbed dynamical system consisting of two ordinary differential equations. This system of equations is close to the system of averaged equations of motion of a rigid body relative to a fixed point [14], obtained taking into account the first two approximations. Indeed, the first equation for the slow variable contains the resonance frequency ratio in the denominator of the right-hand side. Consequently, the conditions presented in the paper can find application in the analysis of the phenomena of strong and weak external stability of resonances in various technical problems close to the problem of the rotation of a perturbed rigid body. In particular, when analyzing the external stability of resonances during disturbed motion relative to the center of mass of spacecraft in the atmospheres of terrestrial planets and small satellites in orbit. In addition, the external stability conditions obtained in this work can be used to analyze the disturbed rotation of gyroscopes with a small mass asymmetry. In this case, it is required that the equations of motion of these technical devices can be written in the form of a system of equations with one fast phase. In addition, after non-resonance averaging on the right-hand side of the equation for a slow frequency, the first approximation should be equal to zero, and the second approximation should be a continuous and differentiable function of constant sign. Moreover, the righthand side of the equation for the fast variable must also be a continuous and differentiable function of constant sign.

It should be noted that the system under consideration has only one slow variable. Such a system of equations describes the motion of a rigid body at small angles of nutation. However, in practice, at considerable nutation angles, it is required to analyze a system of equations with two averaged slow variables. The solution to this more complex problem of 
monotonic external stability of resonance is beyond the scope of this paper, but it is planned to study it in subsequent publications.

\section{Conclusion}

This paper analyzes the monotonic external stability of the resonance in a single perturbed two-frequency nonlinear system consisting of two ordinary differential equations. If the frequencies of the system coincide for a long time, the resonance can be implemented. The main results of this paper are to obtain the conditions of monotonic external stability and instability of this resonance. The author is not aware of publications containing conditions for monotonic external stability of an individual resonance in similar dynamical systems. It should be noted that in this paper were obtained as the necessary conditions, as well as well as the necessary and sufficient conditions for the monotonous external stability and instability of the separate resonance. All the formulated conditions for monotonic external stability and instability of resonance assume that the signs of the analyzed derivatives of the resonance ratio of the frequencies of the first and third orders remain unchanged in the considered non-resonant domain of the change in the time. In addition, this paper gives a new classification of the phenomenon of external monotonic stability of resonance, i.e. weak, linear, and strong stability of the resonance are identified. The linear monotonic external stability of an individual resonance is understood as a monotonous non-resonant convergence of the magnitude of the resonant ratio to the resonance domain by means of linear dependence. The weak nonlinear monotonic external stability of an individual resonance is understood as the monotonic convergence of the magnitude of the resonance ratio to a small resonant domain, which arises in the considered non-resonant domain and occurs over a longer period of time than in the case of linear external stability. The strong nonlinear monotonic external stability of an individual resonance is understood as the monotonic convergence of the ratio of resonances to a small resonance domain, which arises in the considered non-resonant domain and occurs over a shorter period of time than in the case of linear external stability. By analogy, the article introduces a classification of linear, weak and strong monotonic external instability of the resonance.

In general, the mathematical results of the work relate to the study of the influence of resonant denominators on the nonresonant evolution of slow variables of dynamical systems. The author is not aware of publications containing conditions for monotonic external stability of an individual resonance in a system with one fast and one slow variable. These new results can be directly used to analyze the monotonic external stability of resonance in dynamical systems of the type under consideration. It is important to note that some systems of equations averaged in the non-resonant case also reduce to the presented system.

The concluding part of the paper considers the application of the theoretical results obtained within the framework of the problem of the perturbed motion of a rigid body relative to a fixed point. Note that the conditions for monotonic external stability and instability of resonance presented in the paper can be used to analyze the stability of resonances in various modern scientific and technological problems close to the problem of the perturbed rotational motion of the Lagrange top. Such problems, for example, include studies of the rotational motion of an asymmetric gyroscope, the research of motion relative to the center of mass of a descent asymmetric spacecraft in the atmosphere, the analysis of the disturbed rotation of a small satellite relative to its own center of mass, etc.

\section{References}

[1] Ju. Berendsen, M. Burger, V. Ehrlacher, and Jan-Frederik Pietschmann, "Uniqueness of strong solutions and weakstrong stability in a system of cross-diffusion equations," Journal of Evolution Equations, vol. 20, pp. 459-483, Jun. 2020.

[2] Ju. Berendsen, M. Burger, V. Ehrlacher, and Jan-Frederik Pietschmann, Strong solutions and weak-strong stability in a system of cross-diffusion equations, Dec. 2018.

[3] W. Wang, J. Xu, and Xiuting Sun, "Strong and weak resonances in delayed differential systems," International Journal of Bifurcation and Chaos, vol. 23, no. 07, 2013.

[4] Jiang, Heping and Song, Yongli, "Normal forms of nonresonance and weak resonance double Hopf bifurcation in the retarded functional differential equations and applications," Applied Mathematics and Computation, vol. 266, pp. 1102-1126, 2015.

[5] A. I. Mees, "Limit cycle stability, IMA Journal of Applied Mathematics", vol. 11, Issue 3, pp. 281-295, Jun. 1973.

[6] Yeong-Jeu Sun, Yu-Biaw Wu, and Ching-Cheng Wang, "Existence and uniqueness of the exponentially stable limit cycle for a class of nonlinear systems via timedomain approach with differential inequality", Journal of Applied Mathematics, vol. 2013, Article ID 712932, 7 pages, 2013.

[7] A. Ghaffari, M. Tomizuka, and R.A. Soltan, "The stability of limit cycles in nonlinear systems", Nonlinear Dynamics, vol. 56, pp. 269-275, May 2009.

[8] P. Sun, X. Zhao, T. Sun, N. Xu and G. Zong, "Global stability at a limit cycle for switched multi- valued logical networks", Asian journal of control, vol.23, Issue.2, pp.860-870, March 2021.

[9] S. Arroyo, D. H. Zanette, "Duffing revisited: phase-shift control and internal resonance in self-sustained oscillators", The European Physical Journal B, vol. 89, article number 12, Jan. 2016.

[10]D. H. Zanette, "Stability of two-mode internal resonance in a nonlinear oscillator," The European Physical Journal $B$, vol. 91, article number 89, May 2018.

[11]D.H. Zanette, "Effects of noise on the internal resonance of a nonlinear oscillator," Scientific Report, vol. 8, article number 5976, April 2018.

[12] Y.A. Sadov, "Secondary resonance effects in mechanical systems," Mechanics of Solids, no. 4, pp. 20-24, Jul.1990. 
[13] Y.A. Sadov, "Systems with slow evolution and secondary resonance effects," in Materials of the 2nd All-Russian Seminar on Classical and Celestial Mechanics, 1996.

[14] V.V. Lyubimov, "External stability of a resonance in a nonlinear system with slowly changing variables," Mechanics of Solids, vol. 37, no. 6, pp. 42-47, Nov. 2002.

[15] V.V. Lyubimov, "External stability of resonances in the motion of an asymmetric rigid body with a strong magnet in the geomagnetic field," Mechanics of Solids, vol. 45, no. 1, pp. 10-21, Jan. 2010.

[16] V.V. Lyubimov and V.S. Lashin, "External stability of a resonance during the descent of a spacecraft with a small variable asymmetry in the martian atmosphere," Advances in Space Research, vol. 59, pp. 1607-1613, Jan. 2017.

[17] Yu.M. Zabolotnov and V.V. Lyubimov, "Secondary resonance effects during the rotation of a rigid body around a fixed point," Mechanics of Solids, vol. 45, no. 1, pp. 49-59, Jan. 2002.

Vladislav V. Lyubimov received his candidate scientific degree in Physics and Mathematics and doctor scientific degree in Technical Sciences in Samara State Aerospace University, Samara, Russia, in 1998 and 2009, respectively.

He currently serves as the Head of the Chair of Further Mathematics of the Samara National Research University, Samara, Russia.

Research interests: stability of motion of rigid bodies, dynamics of spacecraft flight, perturbation theory, probability theory, number theory.

Vladislav V. Lyubimov is full member of international public association "Academy of navigation and motion control".

\section{Creative Commons Attribution License 4.0 (Attribution 4.0 International, CC BY 4.0)}

This article is published under the terms of the Creative Commons Attribution License 4.0

https://creativecommons.org/licenses/by/4.0/deed.en_US 